\title{
A Retrospective Study of Clinical Presentation of Child with Febrile Seizure and Its Duration
}

\author{
Nipun Shrestha ${ }^{1}$, Dhruba Shrestha ${ }^{1}$, Ashish Shrestha ${ }^{2}$, Mala Shrestha ${ }^{1}$ \\ ${ }^{1}$ Siddhi Memorial Hospital (for Women and Children), Bhaktapur, Nepal \\ ${ }^{2}$ Northampton General Hospital, Northampton, England, United Kingdom
}

Email address:

nipsam@hotmail.com.(N.Shrestha)

\section{To cite this article:}

Nipun Shrestha, Dhruba Shrestha, Ashish Shrestha, Mala Shrestha. A Retrospective Study of Clinical Presentation of Child with Febrile Seizure and Its Duration. American Journal of Pediatrics. Vol. 6, No. 3, 2020, pp. 341-345. doi: 10.11648/j.ajp.20200603.38

Received: May 4, 2020; Accepted: July 22, 2020; Published: August 20, 2020

\begin{abstract}
Background: Febrile Seizure is defined as seizure occurring during febrile episode (body temperature $>100.4^{\circ} \mathrm{F}$ or $38^{\circ} \mathrm{C}$ ) in a child between 6 to 60 months, in absence of CNS infection, metabolic abnormalities, neurological condition and a history of prior afebrile seizure. Some of the major concerns regarding febrile seizure are its duration and recurrence. Risk factors associated with its duration are poorly understood. Method: A retrospective study was conducted from January to December 2018 among children admitted to Siddhi Memorial Hospital (for Women and Children), Bhaktapur (Nepal) with a diagnosis of febrile seizure. Information on those children, including gender, age of onset, type of seizure, duration of seizure etc., were collected from patient records. Children meeting the standard definition of febrile seizure were included in the study. Data analysis was done using Microsoft Excel and STATA, version 15. A p value $<0.05$ was considered statistically significant. Result: A total of 1389 children had been admitted to pediatric department during the study period. Among them, 124 children (i.e. $8.9 \%$ of total admission) met the criteria of febrile seizure and were hence included in the study. Majority of the cases had Simple febrile Seizure $(77 \%)$ and the remaining had Complex febrile seizure $(23 \%)$. The study population was of mixed gender $(60 \%$ male and $40 \%$ female). Mean age of presentation was $23.7( \pm 11.7)$ months. The highest prevalence was seen in children $<2$ years of age. Mean duration of seizure was $5.11( \pm 4.84)$ minutes. URTI and AGE were the leading cause of febrile seizure, with prevalence of $45 \%$ and $42 \%$ respectively. $10 \%$ of children were stunted and $5 \%$ of children were wasted. Mean height for age was $-0.43( \pm 1.5) \mathrm{SD}$ and mean weight for height was $-0.31( \pm 1.02) \mathrm{SD}$. Mean serum sodium, potassium and calcium level were $133( \pm 2.81) \mathrm{meq} / \mathrm{l}, 4.4( \pm 3.0) \mathrm{meq} / \mathrm{l}$ and $8.14( \pm 0.99) \mathrm{mg} / \mathrm{dl}$ respectively and mean random blood sugar was $108.4( \pm 24.17) \mathrm{mg} / \mathrm{dl}$. No association was found between serum sodium level and duration of seizure (correlation=-0.19, $\mathrm{p}=0.832$ ), serum potassium level and duration seizure (correlation $=-0.03, \mathrm{p}=0.7$ ). Positive correlation were found between serum calcium level and duration of seizure (correlation $=+0.25, \mathrm{p}=0.03$ ), as well as blood sugar level and duration of seizure (correlation $=+0.19, \mathrm{p}=0.027$ ). Conclusion: Since blood sugar and serum calcium levels tended to be high among our study subjects, their impact on duration of febrile seizure should be investigated further. Serum sodium and potassium level had no effect on seizure duration.
\end{abstract}

Keywords: Febrile Seizure, Afebrile Seizure, Stunted, Wasted, Sodium, Potassium, Calcium, Blood Sugar, Hyperglycemia, Hyponatremia, Dyselectrolytemia, AGE, URTI, CNS

\section{Introduction}

Febrile Seizure is defined as seizure that occur during febrile episode (body temperature above $100.4^{\circ} \mathrm{F}$ or $38^{\circ} \mathrm{C}$ ) in a child between 6 to 60 months of age, in the absence of Central nervous system (CNS) infection, metabolic disorders, neurological abnormalities and a history of prior afebrile seizures [1]. It is the most common type of seizure in children, accounting for $2-5 \%$ of children of that age group [2] with peak incidence at 18 to 22 months of age [3]. It is classified into two types: simple febrile seizure and complex febrile Seizure [4]. Simple febrile seizure is a generalized seizure that lasts less than 15 minutes and occurs only once in a 24 hour period of fever whereas complex febrile seizure is focal seizure or lasts for more than 15 minutes or occurs more than once in a 24 hour period of fever [5]. Most febrile seizure are simple febrile seizures and 9 to $35 \%$ of febrile 
seizures are complex in nature [4].

Some of the major concerns regarding febrile seizure are its duration and recurrence. There is $30 \%$ chance of recurrence after a first episode simple febrile seizures and $50 \%$ chance of recurrence after 2 or more episodes [2]. Risk factors associated with the recurrence of febrile seizure includes seizure at less than 1 year of age, shorter duration of fever before occurrence of seizure, seizure at lesser degree of fever, history of febrile seizure and epilepsy in first degree and second degree relatives, male child, and child at daycare center [2]. The etiologies of febrile seizure are also highly diverse [6]. There aren't many known risk factors associated with its duration. This study was conducted with the objective evaluating clinical presentation of children with febrile seizures and risk factors associated with duration of febrile seizure among patients in our hospital.

\section{Method}

A retrospective study was conducted among children admitted to the Siddhi Memorial Hospital (for Women and Children) in Bhaktapur, Nepal with a diagnosis of febrile seizure from January 2018 to December 2018. Information on those children, including gender, age of onset, type of seizure, duration of seizure, family history of seizure, were collected from patient records. Children meeting the standard definition of febrile seizure were included in the study. Children below 6 month of age, above 5 years of age, those with afebrile seizure, developmental delay, central nervous system infection like meningitis, were excluded from the study. Data analysis was done using the Microsoft Excel and STATA 15. Statistical significance was inferred if $p<0.05$.

\section{Result}

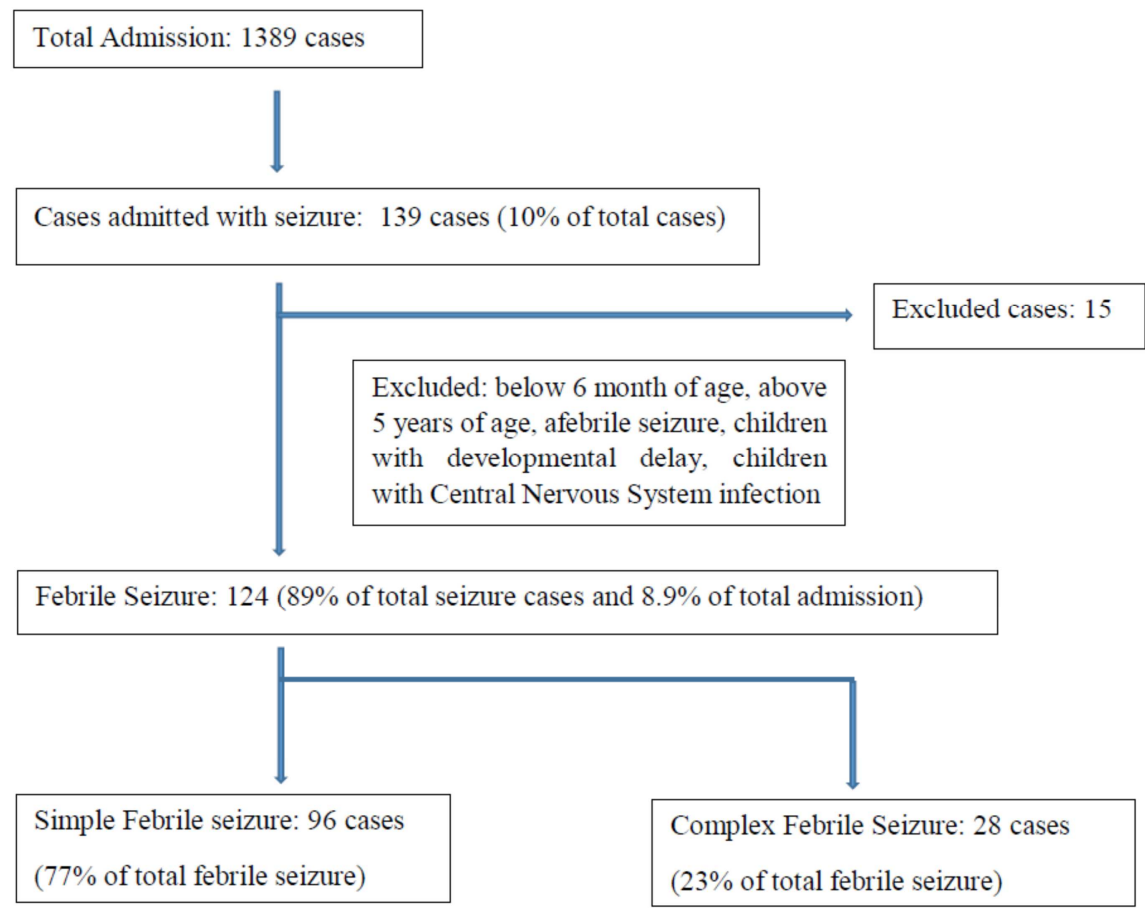

Figure 1. General algorithm of enrolled cases.

Table 1. Age distribution of children with Febrile Seizure.

\begin{tabular}{lll}
\hline Age (months) & Number of cases & Percentage \\
\hline $6-24$ & 71 & 57 \\
$25-48$ & 49 & 40 \\
$49-60$ & 4 & 3 \\
\hline
\end{tabular}

A total of 1389 children were admitted in pediatric departments with diverse diagnosis during the study period. Among them 139 children were admitted with a history of seizure, of which 124 children (i.e. 8.9\% of total admission) met the criteria of febrile seizure and were hence included in the study. Majority of the cases were simple febrile seizure (77\%) and the remaining were Complex febrile seizure (23\%). The study population was of mixed gender $(60 \%$ male and $40 \%$ female). Mean age of presentation was $23.7( \pm 11.7)$ months. The highest prevalence of febrile seizure was seen in children less than 2 years of age (Table 1). A general scheme for inclusion in the study is shown in Figure 1. Mean duration of seizure was $5.11( \pm 4.84)$ minutes. Upper Respiratory Tract Infection (URTI) and Acute Gastroenteritis (AGE) were the leading cause of febrile seizure, with prevalence of $45 \%$ and $42 \%$ respectively (Figure 2). Of all the cases, only 2 needed PICU admission, one child had prolonged duration of seizure and another had a foreign body in the left main bronchus. Prophylactic antibiotic, Inj cefotaxime, was used in $80 \%$ of cases, mainly in cases of 
respiratory tract infection. Symptomatic management was done in $20 \%$ of cases, mainly in cases of acute gastroenteritis. Most of the cases of febrile seizure were seen in March (27 cases), followed by February (16 cases), as presented in Figure 3.

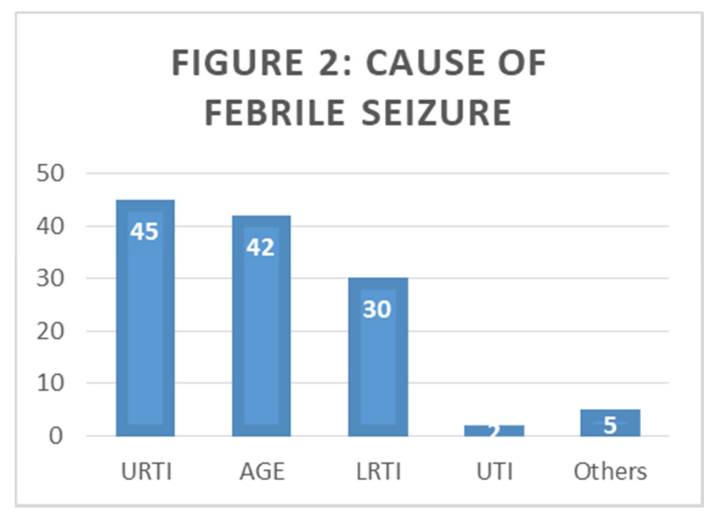

Figure 2. Causes of Febrile Seizure.

We also evaluated Height for Age and Weight for Height of children with febrile seizure. We found that $10 \%$ of children were stunted (height for age below -2SD) and 5\% of children were wasted (weight for height below -2SD). Mean height for age was $-0.43( \pm 1.5) \mathrm{SD}$ and mean Weight for height was $-0.31( \pm 1.02) \mathrm{SD}$.

We evaluated the serum electrolytes, serum calcium and random blood sugar in our all cases as indicated. $68 \%$ of them had some degree of hyponatremia (serum sodium less than $135 \mathrm{mEq} / \mathrm{l})$. Mean serum sodium level was $133( \pm 2.81)$ meq/l. No cases had hypernatremia. Only $4.8 \%$ of cases had hypokalemia and the remaining had normal serum potassium level. Mean serum potassium level was $4.4( \pm 3.0)$ meq/l. Mean serum calcium was $8.14( \pm 0.99) \mathrm{mg} / \mathrm{dl}$ and mean random blood sugar was $108.4( \pm 24.17) \mathrm{mg} / \mathrm{dl}$. Notably, 37\% of cases had random blood sugar level above $120 \mathrm{mg} / \mathrm{dl}$.

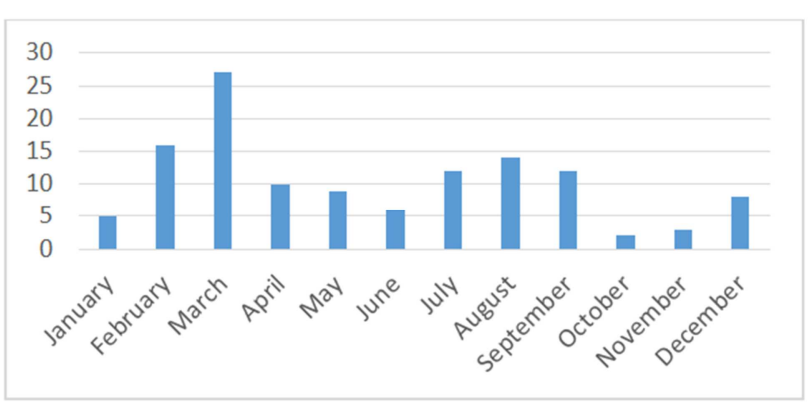

Figure 3. Monthly Admission Pattern.

After careful analysis of the recorded data, we found no association between serum sodium level and duration of seizure (correlation $=-0.19, \mathrm{p}=0.832$ ). Similar results were found for the correlation between serum potassium level and duration of seizure (correlation=-0.03, $\mathrm{p}=0.7$ ). However, a positive correlation was found between serum calcium level and duration of seizure (correlation $=+0.25, \mathrm{p}=0.003$ ). Blood sugar level was also found to be positively correlated with seizure duration (correlation $=+0.19, \mathrm{p}=0.027$ ). These results indicate that the duration of seizure is associated with blood sugar and serum calcium levels in children (Table 2).

Table 2. Association of duration of febrile seizure with serum electrolytes and blood sugar.

\begin{tabular}{llll}
\hline Risk Factor for Duration of Seizure & Mean & Duration of Seizure (min) & P value \\
\hline Serum Sodium $(\mathrm{Na}$ in $\mathrm{meq} / \mathrm{f})$ & $133( \pm 2.81)$ & $5.11( \pm 4.84)$ & 5.832 \\
Serum Potassium $(\mathrm{K}$ in $\mathrm{meq} / \mathrm{l})$ & $4.4( \pm 3.0)$ & $5.11( \pm 4.84)$ & 0.709 \\
Serum Calcium $(\mathrm{Ca}$ in $\mathrm{mg} / \mathrm{dl})$ & $8.14( \pm 0.99)$ & $5.11( \pm 4.84)$ & 0.003 \\
Random Blood Sugar (RBS in $\mathrm{mg} / \mathrm{dl})$ & $108.4( \pm 24.17)$ & 0.027 \\
\hline
\end{tabular}

\section{Discussion}

This study describes the clinical presentation of febrile seizure and risk factors associated with its duration in children. Most cases of febrile seizure were generalized and simple in nature, occurring once every 24 hour and lasting less than 15 minutes. Our observation that most seizures were simple in nature is consistent with that of many other studies [6-8]. Our results also indicate that male children experience more febrile seizure than female children, which has been reported previously [6-8]. The highest prevalence of febrile seizure, in our study, was seen in children less than 2 years of age, consistent with published reports $[6,8]$.

Our study also found that some children with febrile seizure were stunted and wasted. In one study involving 121 children, $52.3 \%$ of children with first episode of febrile seizure had wasting and $76.8 \%$ of children with recurrent seizure had wasting. Additionally, stunting was observed in $21.5 \%$ of the children with first episode of febrile seizure and
$34.7 \%$ of children with recurrent episodes [2]. Malnutrition could therefore be a modifiable risk factor for febrile seizure.

Serum electrolyte levels have always been always been of interest as as a risk factors for febrile seizure [7]. While most studies on febrile seizure have examined the association between electrolyte balance and seizure occurrence, data on how duration of seizure may be related to electrolyte imbalance is scant. Measurement of serum sodium, calcium, phosphorus, magnesium, zinc and iron has always been helpful for clinicians and researchers [9]. It is believed that variations in serum electrolyte levels can increase an individual's susceptibility to develop seizure and its recurrence [10]. Some lines of evidence indicate that hyponatremia may predispose a febrile child to seizure [11]. Lower level of calcium, even if not in hypocalcemic range, is associated with occurrence of febrile seizure [12]. Ionized calcium deficiency has been proposed as a risk factor for febrile seizure [13]. A more comprehensive study is needed to determine if serum calcium level affects febrile seizure duration. Some studies have reported episodes of 
hyperglycemia after an episode of febrile seizure [14]. Stress related events like febrile seizure or high body temperature (above $39^{\circ} \mathrm{C}$ ) have been reported to cause hyperglycemia due to uncoordinated insulin response $[15,16]$. Notably, hyperglycemia is known to induce seizure as well $[17,18]$. Therefore, serum glucose level and occurrence of febrile seizure seems to be interrelated. However, a large systematic investigation is needed to determine if serum glucose level has a causal effect on seizure duration.

\section{Conclusion}

We found positive association of both blood sugar and serum calcium level with duration of febrile seizure. Serum sodium level may affect the occurrence of febrile seizure but its level showed no correlation with seizure duration. Serum potassium level had no influence on seizure duration either. Considering the small sample size of our study, the relationship of recurrence and duration of febrile seizures with dyselectrolytemia should be investigated in larger comprehensive study in future.

\section{Abbreviations}

\author{
AGE: Acute Gastroenteritis \\ RBS: Random Blood Sugar \\ Ca: Calcium \\ CNS: Central Nervous System \\ Inj: Injection \\ K: Potassium \\ LRTI: Lower respiratory Tract Infection \\ $\mathrm{mEq} / \mathrm{l}$ : Milliequivalent per liter \\ min: Minute \\ $\mathrm{mg} / \mathrm{dl}$ : Milligram per deciliter \\ $\mathrm{Na}$ : Sodium \\ PICU: Pediatric Intensive Care Unit \\ SD: Standard Deviation \\ URTI: Upper Respiratory tract Infection
}

\section{References}

[1] AAP. Clinical Practice Guidelines-Febrile Seizures. Guidelines for the neurodiagnostic evaluation of the child with a simple febrile seizure. Pediatrics. 2011; 127: 389-94. 10.1542/peds.2010-3318.

[2] Anisha AN, Kumari P, Elizabeth KE. A prospective study of single versus recurrent episodes of typical febrile seizure among children attending a tertiary care hospital. Indian Journal of Child Health [Internet]. \&nbsp; [cited 24 Jun. 2020]; 5 (7): 484-7.

[3] American Academy of Pediatrics Steering Committee on Quality Improvement and Management, Subcommittee on Febrile Seizures. Febrile seizures: Clinical practice guideline for the long-term management of the child with simple febrile seizures. Pediatrics. 2008; 121: 1281-6.
[4] Karande S. Febrile seizures: A review for family physicians, India. J Med sci. 2007; 61 (3): 161-72.

[5] Practice parameter: The neurodiagnostic evaluation of the child with a first simple febrile seizure. American Academy of Pediatrics. Provisional Committee on Quality Improvement, Subcommittee on Febrile Seizures. Pediatrics, 1996; 97: 76972 .

[6] Shrestha D, Dhakal AK, Shakya H, Shakya A, Shah SC, Mehata S. Clinical Characteristics of Children with Febrile Seizure. J Nepal Health Res Counc. 2014; 12 (28): 162-6.

[7] Salehiomran MR, Ebrahimzadeh H, Hajiahmadi M. The serum sodium levels and recurrence of simple febrile seizure during the first 24 hours in children. Caspian J Pediatr. 2018; 4 (1): 278-81.

[8] Veisani Y, Delpisheh A, Sayehmiri K. Familial History and recurrence of febrile seizure: a systemic review and meta analysis. Iran J Pediatr. 2013; 23 (4): 389-395.

[9] Kiviranta T, Airaksinen E. Low sodium levels in serum are associated with subsequent febrile seizures. Acta Paediatr. 1995; 84 (12): 1372-4.

[10] Chiarelli F, De CP, Verrotti A, et al. Electrolytic changes in febrile convulsions. Med Surg Pediatr. 1985; 7 (2): 249-52.

[11] Heydarian F, Ashrafzadeh F, Kam S. Simple febrile seizure: The role of serum sodium levels in prediction of seizure recurrence during the first 24 hours. Iran J Child Neurology. 2009; 3 (2): 31-34. 10.22037/ijcn.v3i2.1269.

[12] Ushakiran CB, Suresh R. Reduced serum calcium is a risk factor for febrile seizures. Int J Contemp Pediatr. 2017. 4 (4): 1506-1508. 10.18203/2349-3291.ijcp20172695.

[13] Sharma S, singh N, Thimmaraju K, Tilak M. ASSESSMENT OF IONIZED CALCIUM STATUS IN FEBRILE SEIZURES Int. j. clin. biomed. res. [Internet]. 31 Jul. 2018 [cited 24 Jun. 2020]; 4 (3): 35-7. Availablefrom: https://sumathipublications.com/index.php/ijcbr/article/view/2 13https://doi.org/10.31878/ijcbr.2018.43.08.

[14] Ghobadifar MA, Honar H, Jooya P, Hassani F. Blood glucose level after febrile convulsion. Korean J Pediatr. 2016, 59 (3): 153-154. 10.3345/kjp.2016.59.3.153.

[15] Valerio G, Franzese A, Carlin E, Pecile P, Perini R, Tenore A. High prevalence of stress hyperglycaemia in children with febrile seizures and traumatic injuries. Acta Paediatr. 2001; 90 (6): 618-22.

[16] Saz EU, Ozen S, Simsek Goksen D, Darcan S. Stress hyperglycemia in febrile children: relationship to prediabetes. Minerva Endocrinol. 2011; 36 (2): 99-105.

[17] Maccario M, Messis CP, Vastola EF. Focal seizures as a manifestation of hyperglycemia without ketoacidosis. A report of seven cases with review of the literature. Neurology. 1965; 15 (3): 195-206. 10.1212/wnl.15.3.195.

[18] Wang X. Nonketotic hyperglycemia-related epileptic seizures. Chinese Neurosurgical Journal. 2017; 3 (1): 16. https://doi.org/10.1186/s41016-017-0073-8. 


\section{Biography}

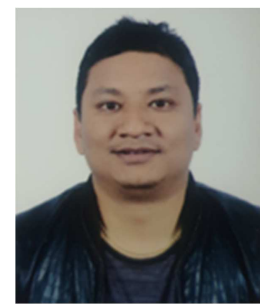

Nipun Shrestha Specialization: MD Pediatrics Rank: Senior Registrar Pediatrician Place of Work: Siddhi Memorial Hospital (for women and children).

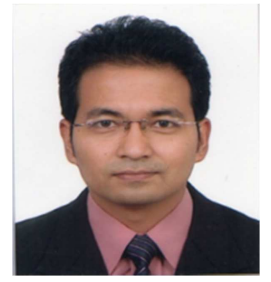

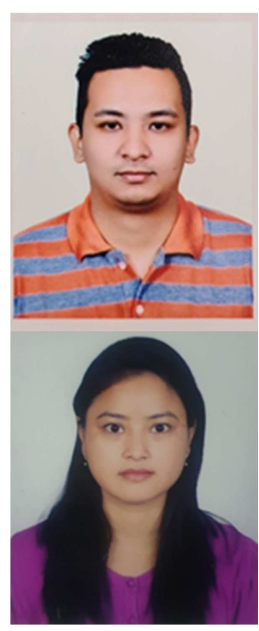

Ashish Shrestha Rank: Senior House Officer Place of Work: Northampton General Hospital.

Mala Shrestha Specialization: MD Obstetrics and Gynaecology Rank: Obstetrician and Gynaecologist Place of Work: Siddhi Memorial Hospital (for women and children). 